\title{
Contact Aspiration versus Stent-Retriever Thrombectomy for Distal Middle Cerebral Artery Occlusions in Acute Ischemic Stroke: Meta-Analysis
}

\author{
Kevin Phan, $\mathrm{MD}^{1,2}$, Julian Maingard, $\mathrm{MBBS}^{3,4}$, Hong Kuan Kok, $\mathrm{MD}^{5}$, Adam A Dmytriw, MD, MSc ${ }^{6}$, \\ Sourabh Goyal, BS ${ }^{1}$, Ronil Chandra, $\mathrm{MBBS}^{7}$, Duncan Mark Brooks, MBBS ${ }^{3,4}$, Vincent Thijs, $\mathrm{MD}^{8,9}$, \\ Hamed Asadi, $\mathrm{MD}^{3,7}$ \\ ${ }^{1}$ NeuroSpine Surgery Research Group, Prince of Wales Private Hospital, Randwick, Australia \\ ${ }^{2}$ Department of Neurosurgery, Prince of Wales Hospital, Randwick, Australia \\ ${ }^{3}$ Interventional Neuroradiology Service, Department of Radiology, Austin Hospital, Heidelberg, Australia \\ ${ }^{4}$ Department of Radiology, School of Medicine, Faculty of Health, Deakin University, Waurn Ponds, Australia \\ ${ }^{5}$ Department of Interventional Radiology, Guy's and St Thomas' NHS Foundation Trust, London, UK \\ ${ }^{6}$ Department of Radiology, University of Toronto, Toronto, Canada \\ ${ }^{7}$ Interventional Neuroradiology Unit, Monash Imaging, Monash Health, Clayton, Australia \\ ${ }^{8}$ Stroke Division, Florey Institute of Neuroscience and Mental Health, University of Melbourne, Parkville, Australia \\ ${ }^{9}$ Department of Neurology, Austin Health, Heidelberg, Australia
}

Purpose: The evidence for endovascular therapy and choice of technique in distal middle cerebral artery (MCA) M2 segment occlusions in acute ischemic stroke remains controversial. We aimed to conduct a systematic review and meta-analysis primarily comparing reperfusion rates of stent-retrieval versus contact aspiration for M2 occlusions.

Materials and Methods: Study selection included cohorts of patients with distal MCA occlusions in acute ischemic strokes treated with an endovascular approach including stent-retrieval or contact aspiration. Twelve studies were selected for meta-analysis for a total of 835 cases. Meta-analysis by proportions was conducted on parameters including baseline and procedural characteristics, thrombolysis in cerebral infarction (TICl) 2b-3 outcomes, and 90-day modified Rankin scale (mRS) outcomes

Results: Hypertension and hyperlipidemia were more prevalent in stent-retriever patients. Pooled baseline National Institute of Health Stroke Scale scores and Alberta Stroke Program Early Computed Tomography Score imaging scores were similar. Pooled time onset of symptoms to door arrival was higher for the stent-retrieval group (154 vs. 97.4 minutes, $P=0.01$ ), as was time to groin puncture (259.9 vs. 156.2 minutes, $P=0.02$ ), but there was no difference in procedure time. The $\mathrm{TICl} 2 \mathrm{~b}-3$ recanalization rate was similar ( $80.5 \%$ vs. $86.8 \%, \mathrm{P}=0.168)$. The frequency of mRS 0-2 at 90-day was also similar (74.5\% vs. 59.9\%, $\mathrm{P}=0.120)$, and an excellent mRS 0-1 was lower for stent-retrievers (39.9\% vs. 65.6\%, P=0.003). A significant negative correlation was found between onset to groin puncture time and the proportion of patients with a good $m R S(r=-0.71, P=0.048)$.

Conclusion: Both endovascular techniques achieved recanalization rates greater than $80 \%$ and 90-day outcomes of minimal disability with similar complication rates. The literature is skewed by aspiration cases being performed sooner after onset of stroke compared to stent-retriever cases.

Key Words: Cerebrovascular disorders; Ischemia; Stroke; Thrombectomy; Endovascular procedures; Middle cerebral artery

\author{
Correspondence to: \\ Hamed Asadi, MD \\ Interventional Neuroradiology Ser- \\ vice, Department of Radiology, Austin \\ Hospital, 145 Studley Rd, Heidelberg \\ VIC 3084, Australia \\ Tel: +61-3-9496-5625 \\ Fax: +61-3-9459-2817 \\ E-mail: asadi.hamed@gmail.com
}

Received: April 25, 2018

Revised: July 19, 2018

Accepted: August 9, 2018

Copyright $\odot 2018$ Korean Society of Interventional Neuroradiology

This is an Open Access article distributed under the terms of the Creative Commons Attribution Non-Commercial License (http://creativecommons.org/licenses/by-nc/3.0) which permits unrestricted non-commercial use, distribution, and reproduction in any medium, provided the original work is properly cited. 


\section{INTRODUCTION}

With recent overwhelming level 1 evidence ${ }^{1-7}$ for the efficacy and safety of mechanical thrombectomy over best medical management for large vessel occlusion acute ischemic strokes, there has been a shift in research priorities towards optimizing time to recanalization, imaging protocols, risk stratification and selection criteria, and development and assessment of newer thrombectomy techniques. One such challenge to be addressed is understanding the effectiveness and role of mechanical thrombectomy in patients with more distal vessel occlusions, and whether results in proximal large vessel occlusions can be extrapolated to this population or not.

For occlusions of the second part of the middle cerebral artery (MCA, M2 segment) and more distally, the occluded lumen is often smaller with thinner walls, which may be more technically challenging to access with a catheter and for stent-retriever deployment. As such, there is theoretically an increased risk of complications, such as dissection and perforation, for endovascular interventions in this area. Furthermore, the efficacy profile of mechanical thrombectomy in $\mathrm{M} 2$ and more distal occlusions may also differ. The ischemic penumbra volume of distal occlusions tends to be smaller and can also lessen the benefit of endovascular thrombectomy and may be more amenable to intravenous thrombolysis therapy. ${ }^{8,9}$ However, there is also the risk that important eloquent areas can be affected despite limited vascular territory involvement. As such, endovascular therapy for M2 occlusions remains controversial.

Current American Heart Association and Stroke Association guidelines ${ }^{10}$ support the use of mechanical thrombectomy for distal occlusions in selected patients within 6 hours of symptom onset. This can involve stent-retrieval or new aspiration first approach or A Direct Aspiration First Pass Technique (ADAPT) approach with large bore aspiration catheters for thrombectomy. ${ }^{11-14}$ The ADAPT technique has potential benefits of a high rate of complete revascularization, reduced groin puncture to revascularization time, a lesser technical difficulty compared to stent-retrievers, and increased cost effectiveness. ${ }^{11-14}$ There has been a prior systematic review ${ }^{15}$ exploring the role of endovascular therapy in $\mathrm{M} 2$ occlusion strokes, reporting results from eight studies with overall successful reperfusion in 78\% of cases and 90 -day good mRS rates of $62 \%$, with acceptable mortality and complication rates. However, there is limited literature directly compar- ing these two contemporary mechanical thrombectomy options in the context of endovascular interventions for $\mathrm{M} 2$ occlusion strokes. Therefore, we conducted a systematic review and meta-analysis to compare reperfusion rates and complications of stent-retrieval versus contact aspiration for treating M2 segment occlusions and determine factors which correlate with favorable clinical outcome.

\section{MATERIALS AND METHODS}

\section{Literature search strategy}

The present study was conducted according to Preferred Reporting Items for Systematic Reviews and Meta-Analyses and recommended guidelines for systematic reviews. ${ }^{16}$ Systematic searches of electronic databases were performed using Ovid Medline, PubMed, Cochrane Central Register of Controlled Trials (CCTR), Cochrane Database of Systematic Reviews (CDSR), ACP Journal Club, and Database of Abstracts of Review of Effectiveness (DARE) from their dates of inception to September 2017. The search strategy entailed combination of the terms "stroke", "middle cerebral artery", "MCA", "thrombectomy", "endovascular", "thrombectomy", "ADAPT", or "FAST". The reference lists of all retrieved articles were reviewed for further identification of potentially relevant studies and assessed using the inclusion and exclusion criteria.

\section{Selection criteria}

Eligible studies for the present systematic review and meta-analysis included cohorts of patients with distal MCA occlusion acute ischemic stroke that were treated with an endovascular approach including stent-retrieval technology or contact aspiration. All studies which reported outcome for M2 and distal MCA occlusions (regardless of specific anatomical or territorial definition) were included for consideration for analysis. However, the M2 segment was generally defined as vertical MCA branches originating at the genu to the apex of the circular sulcus. Included studies must have reported either thrombolysis in cerebral infarction (TICl) 2b-3 outcomes or modified Rankin scale (mRS) outcomes at follow-up. ${ }^{17}$

Studies which had fewer than 10 patients with acute M2 occlusions were excluded. Studies published before January 1, 2015 were excluded in order to capture modern thrombectomy results. When institutions published duplicate studies with accumulating numbers of patients or increased lengths of follow-up, only the most complete reports were 
included for quantitative assessment at each time interval. All publications were limited to those involving human subjects and in the English language. Abstracts, case reports, conference presentations, editorials, reviews, and expert opinions were excluded.

\section{Data extraction}

All data were extracted from article texts, tables, and figures by two reviewers (K.P. and J.M.) and discrepancies were discussed via consensus. If a study provided medians and interquartile ranges instead of means and standard deviations (SDs), we imputed the means and SDs as described by Hozo et al. ${ }^{18}$ Demographic data studied included year, study period, number of patients, age, gender, hypertension, hyperlipidemia, diabetes, atrial fibrillation, prior stroke/transient ischemic attack (TIA), current smoker, treatment window, National Institute of Health Stroke Scale (NIHSS) score at presentation, Alberta Stroke Program Early Computed Tomography Score (ASPECTS), and use of intravenous tissue plasminogen activator (IV-tPA).Treatment parameters included thrombectomy technique, successful reperfusion/recanalization of TICl $2 b-3$, onset to door time, onset to groin puncture time, time of groin puncture to recanalization. Outcome data included mRS 0-2 (good) and mRS 0-1 (excellent) at 90-day follow-up, mortality, total intracerebral hemorrhage (ICH), symptomatic $\mathrm{ICH}(\mathrm{s} \mid \mathrm{CH})$, asymptomatic $\mathrm{ICH}(\mathrm{alCH})$, and procedural complications including perforation, dissection, and distal embolization.

\section{Statistical analysis}

A meta-analysis of proportions was conducted for the available main perioperative and postoperative variables, using logit transformation and combined using DerSimonian-Liard random effects models. Heterogeneity was evaluated using Cochran $\mathrm{Q}$ and $\mathrm{I}^{2}$ test. Heterogeneity was defined as none $\left(I^{2}=0 \%\right)$, low $\left(I^{2}=25 \%\right)$, moderate $\left(I^{2}=50 \%\right)$, or high $\left(I^{2}=75 \%\right){ }^{19}$ A formal statistical comparison was performed between stent-retrieval versus contact aspiration techniques using mixed-effects meta-regression with a moderator variable for thrombectomy technique. Weighted meta-regression analysis was performed to determine whether baseline factors could be associated with outcome and hence be potential confounders. All analyses were performed using the metafor package for $\mathrm{R}$ version 3.01. $\mathrm{P}$ values $<0.05$ were considered statistically significant.

\section{RESULTS}

\section{Search strategy}

A total of 461 articles were identified through the electronic database searches and from other sources including reference lists (Fig. 1). After exclusion of duplicate or irrelevant references, 54 potentially relevant articles were retrieved. After detailed evaluation, 12 studies $8,9,20-30$ were included for assessment (Supplementary Table 1), including 835 cases of distal occlusions of the MCA (predominantly M2 occlusions). Results for contact aspiration were reported in 6 studies $9,20,21,24,25,30$ whilst stent-retrieval outcomes were reported in 10 studies..$^{8,9,21-24,26-29}$ Out of the 835 included cases, there were 223 patients in the contact aspiration group and 612 patients in the stent-retrieval group. All studies were observational in nature except for the Contact Aspiration Versus Stent Retriever for Successful Revascularization (ASTER) randomized trial; ${ }^{21}$ however, M2 occlusions constituted only a subgroup of patients of this study. Definitions of M2 occlusions for each included study are summarized in Supplementary Table 2.

\section{Baseline characteristics}

Overall, patients were elderly with mean age of 69.9 years, which was not significantly different between stent-retriever vs contact aspiration cohorts. There were no differences in the proportions of females between stent-retriever and aspiration cohorts. Hypertension was more prevalent in stent-retriever patients (68.5\% vs. 54.3\%, $\mathrm{P}=0.047)$ as was hyperlipidemia ( $45.4 \%$ vs. $19.6 \%, \mathrm{P}=0.015)$. There were no differences between the groups in terms of diabetes, atrial fibrillation, coronary artery disease, prior stroke/TIA, current smoking, or IV-tPA use. Pooled baseline NIHSS scores and ASPECTS imaging scores were also similar between stent-retriever and contact aspiration groups (Table 1).

\section{Treatment characteristics}

Pooled time onset of symptoms to door arrival was significantly higher for the stent-retrieval group compared to contact aspiration (154 vs. 97.4 minutes, $\mathrm{P}=0.01$ ), as was time to groin puncture (259.9 vs. 156.2 minutes, $P=0.021$ ). There were no significant differences in time from groin puncture to recanalization (35.6 vs. 40.8 minutes, $P=0.273$ ).

\section{Clinical outcomes}

The primary outcome, the proportion of $\mathrm{TICl} 2 \mathrm{~b}-3$ recanali- 


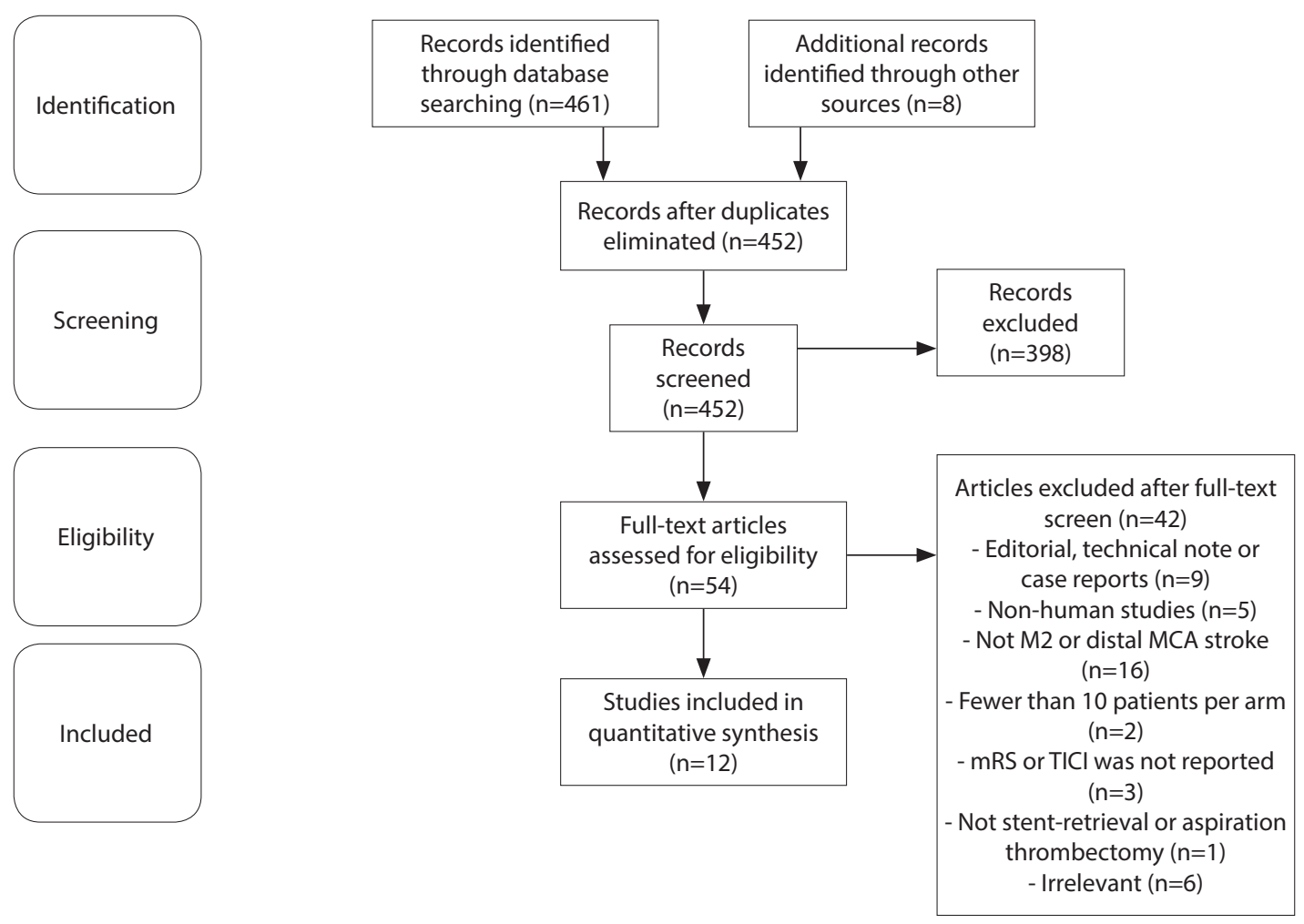

Fig. 1. PRISMA flow chart of search strategy for the present systematic review and meta-analysis. PRISMA, Preferred Reporting Items for Systematic Reviews and Meta-Analyses; MCA, middle cerebral artery; mRS, modified Rankin scale; TICl, thrombolysis in cerebral infarction.

Table 1. Baseline and procedural characteristics

\begin{tabular}{|c|c|c|c|c|}
\hline Variable & Overall & Stent-retriever & Aspiration first & P-value for difference \\
\hline Age (years) & $69.9(67.5-72.3)$ & $69.8(66.1-73.5)$ & $70.7(69.4-72.0)$ & 0.931 \\
\hline Females (\%) & $45.7(40.8-50.6)$ & $46.2(40.4-52.1)$ & $43.9(33.8-54.4)$ & 0.518 \\
\hline Hypertension (\%) & $65.3(57.0-72.8)$ & $68.5(60.3-75.7)$ & $54.3(41.3-66.8)$ & 0.047 \\
\hline Hyperlipidaemia (\%) & $37.0(24.9-51.0)$ & $45.4(31.4-60.2)$ & $19.6(11.2-32.1)$ & 0.015 \\
\hline Diabetes (\%) & $21.5(18.0-25.5)$ & $22.3(18.0-27.2)$ & $16.1(8.6-28.3)$ & 0.314 \\
\hline Atrial fibrillation (\%) & $41.0(32.0-50.7)$ & $40.8(33.6-48.5)$ & $29.0(2.9-84.9)$ & 0.473 \\
\hline Coronary artery disease (\%) & $11.4(5.5-22.0)$ & $13.2(5.6-28.0)$ & $8.0(2.0-26.9)$ & 0.527 \\
\hline Prior stroke/TIA (\%) & $13.1(8.2-20.3)$ & $12.1(6.2-22.4)$ & $14.1(7.2-25.8)$ & 0.742 \\
\hline Current smoker & $14.4(6.7-28.5)$ & $11.2(3.6-29.7)$ & $23.1(13.9-35.9)$ & 0.224 \\
\hline Alteplase use & $58.2(47.5-68.1)$ & $57.5(47.0-67.3)$ & $66.1(33.4-88.3)$ & 0.690 \\
\hline NIHSS baseline & $13.6(12.4-14.9)$ & $13.6(11.9-15.3)$ & $13.8(11.9-15.7)$ & 0.857 \\
\hline ASPECTS baseline & $8.7(8.3-9.1)$ & $8.8(8.3-9.3)$ & $8.5(7.5-9.5)$ & 0.682 \\
\hline Onset to door time (minutes) & $129.1(70.6-187.6)$ & $154.0(146.0-162.0)$ & $97.4(22.0-172.8)$ & 0.010 \\
\hline Onset to groin puncture time (minutes) & $220.9(110.3-331.5)$ & $259.9(229.2-290.6)$ & $156.2(5.3-307.1)$ & 0.021 \\
\hline Time of groin puncture to recanalization (minutes) & $37.5(32.3-42.7)$ & $35.6(29.6-41.7)$ & $40.8(26.0-55.6)$ & 0.273 \\
\hline
\end{tabular}

Values are presented as either weighted mean ( $95 \%$ confidence interval).

TIA, transient ischemic attack; NIHSS, National Institute of Health Stroke Scale; ASPECTS, Alberta Stroke Program Early Computed Tomography Score. 
Studies

Altenbernd et al. ${ }^{20}$

ASTER trial (ADAPT)

Kim et al. ${ }^{9}$ (ADAPT)

Mokin et al. ${ }^{24}$ (ADAPT)

Park and $\mathrm{Kwak}^{25}$

Vargas et al. ${ }^{30}$

Subgroup ADAPT $\left(I^{2}=49.63 \%, P=0.077\right)$

ASTER trial (stent-retriever)

Coutinho et al. ${ }^{22}$

Dorn et al. ${ }^{8}$

Flores et al. ${ }^{23}$

Kim et al. ${ }^{9}$ (stent-retriever)

Mokin et al. ${ }^{24}$ (stent-retriever)

Protto et al. ${ }^{26}$

Salahuddin et al. ${ }^{27}$

Sarraj et al. ${ }^{28}$

Subgroup stent-retriever $\left(\mathrm{I}^{2}=17.87 \%, \mathrm{P}=0.284\right)$

Overall $\left(\mathrm{I}^{2}=36.98 \%, \mathrm{P}=0.074\right)$

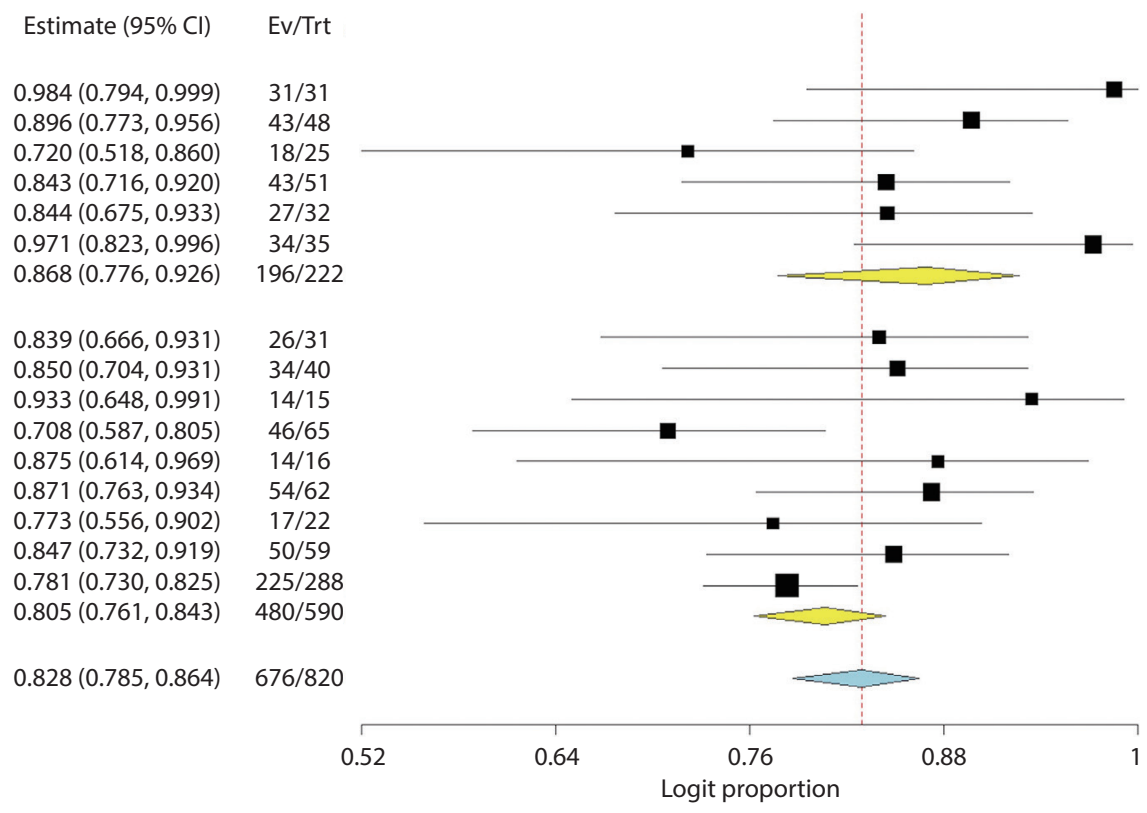

Fig. 2. Forest plot compares pooled outcomes of stent-retriever versus contact aspiration for $\mathrm{TICl} 2 \mathrm{~b}-3$ (P-value for difference 0.168 ). Cl, confidence interval; Ev, events; Trt, treatment; ASTER, Contact Aspiration Versus Stent Retriever for Successful Revascularization; ADAPT, A Direct Aspiration First Pass Technique; $\mathrm{TICl}$, thrombolysis in cerebral infarction.

Table 2. Meta-analysis of procedural and 90-day outcomes

\begin{tabular}{lllll}
\hline Variable & Overall & Stent-retriever & Aspiration first & P-value for difference \\
\hline TICl 2b-3 (\%) & $82.8(78.5-86.4)$ & $80.5(76.1-84.3)$ & $86.8(77.6-92.6)$ & 0.168 \\
\hline TICl 3 (\%) & $55.8(47.0-64.3)$ & $53.5(42.9-63.9)$ & $57.1(43.3-69.9)$ & 0.700 \\
\hline 90-day mRS 0-2 (\%) & $62.0(55.8-67.8)$ & $59.9(55.8-63.8)$ & $74.5(56.4-86.8)$ & 0.120 \\
90-day mRS 0-1 (\%) & $44.2(33.7-55.2)$ & $39.9(31.5-49.0)$ & $65.6(47.9-79.8)$ & 0.003 \\
90-day mortality (\%) & $12.4(8.9-17.0)$ & $14.7(11.0-19.4)$ & $4.3(1.2-14.5)$ & 0.212 \\
All ICH (\%) & $11.2(8.5-14.5)$ & $11.7(8.6-15.6)$ & $9.4(5.0-16.8)$ & 0.530 \\
sICH (\%) & $5.3(3.7-7.5)$ & $5.7(3.9-8.3)$ & $2.6(0.8-7.7)$ & 0.183 \\
alCH (\%) & $6.0(4.2-8.3)$ & $6.6(4.1-10.5)$ & $4.1(1.6-9.9)$ & 0.378 \\
Distal embolization (\%) & $5.7(1.8-17.2)$ & $5.9(0.6-39.4)$ & $4.6(0.9-20.0)$ & 0.598 \\
Dissection or perforation (\%) & $3.3(1.7-6.5)$ & $3.8(1.6-8.7)$ & $1.9(0.5-7.2)$ & 0.344 \\
\hline
\end{tabular}

$\mathrm{TICl}$, thrombolysis in cerebral infarction scale; mRS, modified Rankin scale; ICH, intracerebral hemorrhage; slCH, symptomatic intracerebral hemorrhage; alCH, asymptomatic intracerebral hemorrhage.

zation, was similar for stent-retrievers and contact aspiration (80.5\% vs. 86.8\%, $P=0.168$ ) (Fig. 2). This was associated with a moderate level of heterogeneity $\left(\mathrm{I}^{2}=36.98 \%\right.$ overall). TICl 3 was also comparable (53.5\% vs. 57.1\%, P=0.70). The secondary outcome, mRS 0-2 at 90-day follow-up, was similar for stent-retrievers and contact aspiration (59.9\% vs. 74.5\%, $\mathrm{P}=0.120$ ) (Table 2, Fig. 3). This was associated with a significant level of heterogeneity $\left(I^{2}=51.66 \%\right.$ overall). Excellent mRS at 90 -days was also significantly lower for stent-retrievers
(39.9\% vs. 65.6\%, $P=0.003$ ). No differences were noted in terms of 90 -day mortality (14.7\% vs. $4.3 \%, \mathrm{P}=0.177$ ) (Fig. 4) with a fair level heterogeneity $\left(I^{2}=23.58 \%\right)$, any $\mathrm{ICH}(11.7 \%$ vs. 9.4\%, $\mathrm{P}=0.53)$, slCH (5.7\% vs. 2.6\%, $\mathrm{P}=0.183)$ with low heterogeneity $\left(\mathrm{I}^{2}=0 \%\right)$, or alCH (6.6\% vs. $\left.4.1 \%, \mathrm{P}=0.378\right)$. There were no significant differences in procedural complications between stent-retrieval and aspiration first techniques in terms of distal embolization (5.9\% vs. 4.6\%, $\mathrm{P}=0.598$ ) or dissection/ perforation (3.8\% vs. $1.9 \%, \mathrm{P}=0.344)$. 
Studies

Altenbernd et al. ${ }^{20}$

Kim et al. ${ }^{9}$ (ADAPT)

Mokin et al. ${ }^{24}$ (ADAPT)

Park and $\mathrm{Kwak}^{25}$

Vargas et al. ${ }^{30}$

Subgroup ADAPT $\left(I^{2}=76.49 \%, \mathrm{P}=0.002\right)$

Coutinho et al. ${ }^{22}$

Dorn et al. ${ }^{8}$

Flores et al. ${ }^{23}$

Kim et al. ${ }^{9}$ (stent-retriever)

Mokin et al. ${ }^{24}$ (stent-retriever)

Protto et al. ${ }^{26}$

Salahuddin et al. ${ }^{27}$

Sarraj et al. ${ }^{28}$

Subgroup stent-retriever $\left(I^{2}=0 \%, P=0.540\right)$

Overall $\left(I^{2}=51.66 \%, P=0.016\right)$

$\begin{array}{lr}\text { Estimate }(95 \% \mathrm{Cl}) & \text { Ev/Trt } \\ & \\ 0.968(0.804,0.995) & 30 / 31 \\ 0.840(0.643,0.939) & 21 / 25 \\ 0.529(0.394,0.661) & 27 / 51 \\ 0.781(0.607,0.892) & 25 / 32 \\ 0.594(0.419,0.747) & 19 / 32 \\ 0.745(0.564,0.868) & 122 / 17 \\ & \\ & \\ 0.600(0.460,0.725) & 30 / 50 \\ 0.600(0.348,0.808) & 9 / 15 \\ 0.508(0.388,0.626) & 33 / 65 \\ 0.750(0.492,0.903) & 12 / 16 \\ 0.597(0.471,0.711) & 37 / 62 \\ 0.500(0.302,0.698) & 11 / 22 \\ 0.559(0.432,0.680) & 33 / 59 \\ 0.628(0.571,0.682) & 181 / 288 \\ 0.599(0.558,0.638) & 346 / 577\end{array}$

$0.620(0.558,0.678)$

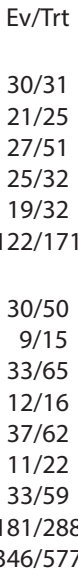

$468 / 748$

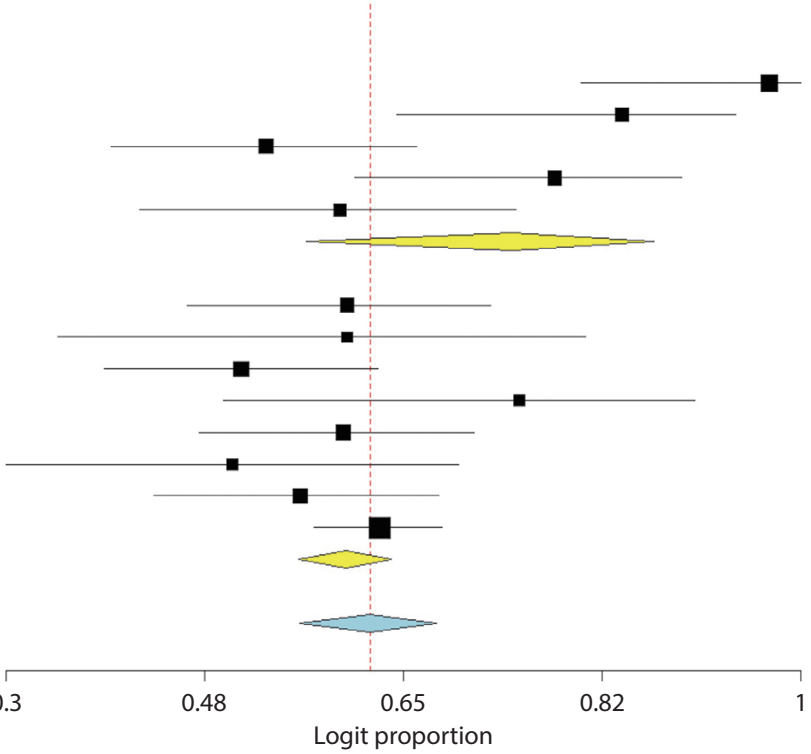

Fig. 3. Forest plot compares pooled outcomes of stent-retriever versus contact aspiration for 90-day mRS 0-2 (P-value for difference 0.041). Cl, confidence interval; Ev, events; Trt, treatment; ADAPT, A Direct Aspiration First Pass Technique; mRS, modified Rankin scale.

Studies

Altenbernd et al..$^{20}$

Kim et al. ${ }^{9}$ (ADAPT)

Mokin et al. ${ }^{24}$ (ADAPT)

Park and $\mathrm{Kwak}^{25}$

Vargas et al. ${ }^{30}$

Subgroup ADAPT $\left(\mathrm{I}^{2}=53.94 \%, \mathrm{P}=0.070\right)$

Coutinho et al. ${ }^{22}$

Dorn et al. ${ }^{8}$

Flores et al. ${ }^{23}$

Kim et al. ${ }^{9}$ (stent-retriever)

Mokin et al. ${ }^{24}$ (stent-retriever)

Protto et al. ${ }^{26}$

Salahuddin et al. ${ }^{27}$

Subgroup stent-retriever $\left(I^{2}=0 \%, P=0.692\right)$

Overall $\left(I^{2}=23.58 \%, P=0.212\right)$
Estimate $(95 \% \mathrm{Cl}) \quad \mathrm{Ev} / \mathrm{Trt}$

$0.016(0.001,0.206)$

$0.019(0.001,0.244)$

$0.157(0.080,0.284)$

$0.031(0.004,0.191)$

$0.014(0.001,0.187)$

$0.043(0.012,0.145)$

$0.120(0.055,0.242)$

$0.067(0.009,0.352)$

$0.138(0.074,0.245)$

$0.063(0.009,0.335)$

$0.210(0.126,0.328)$

$0.136(0.045,0.348)$

$0.136(0.069,0.248)$

$0.147(0.110,0.194)$

$0.124(0.089,0.170)$

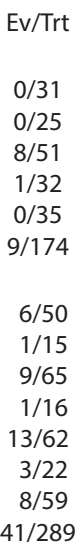

$50 / 463$

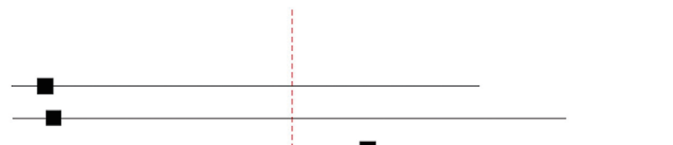

$+$
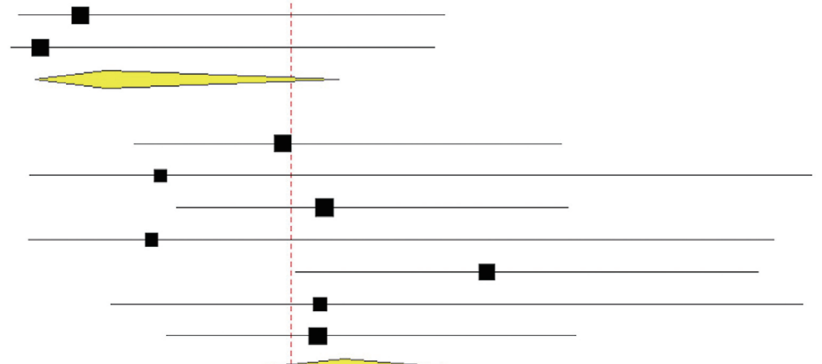

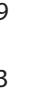

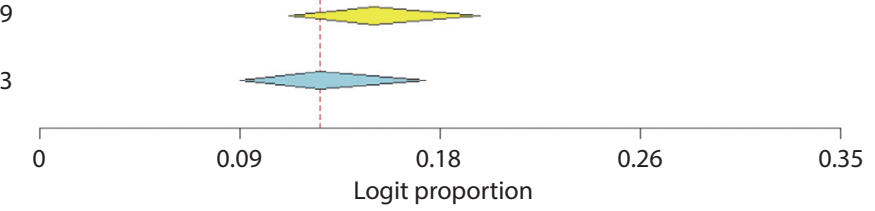

Fig. 4. Forest plot compares pooled outcomes of stent-retriever versus contact aspiration for 90-day mortality (P-value for difference 0.212). Cl, confidence interval; Ev, events; Trt, treatment; ADAPT, A Direct Aspiration First Pass Technique.

\section{Correlation analysis}

In order to investigate potential contributing or confounding factors driving differences in clinical outcomes between stent-retrieval versus aspiration techniques, we performed correlation analysis using baseline variables that were signifcantly different. Significant negative correlation was found between onset to groin puncture time and the proportion of patients with a good mRS ( $r=-0.71, P=0.048)$. However, no significant correlations were found for onset to door time, hypertension, or hyperlipidemia with a good mRS clinical outcome (Fig. 5).

\section{DISCUSSION}

In this study, the efficacy of both stent-retrieval and con- 

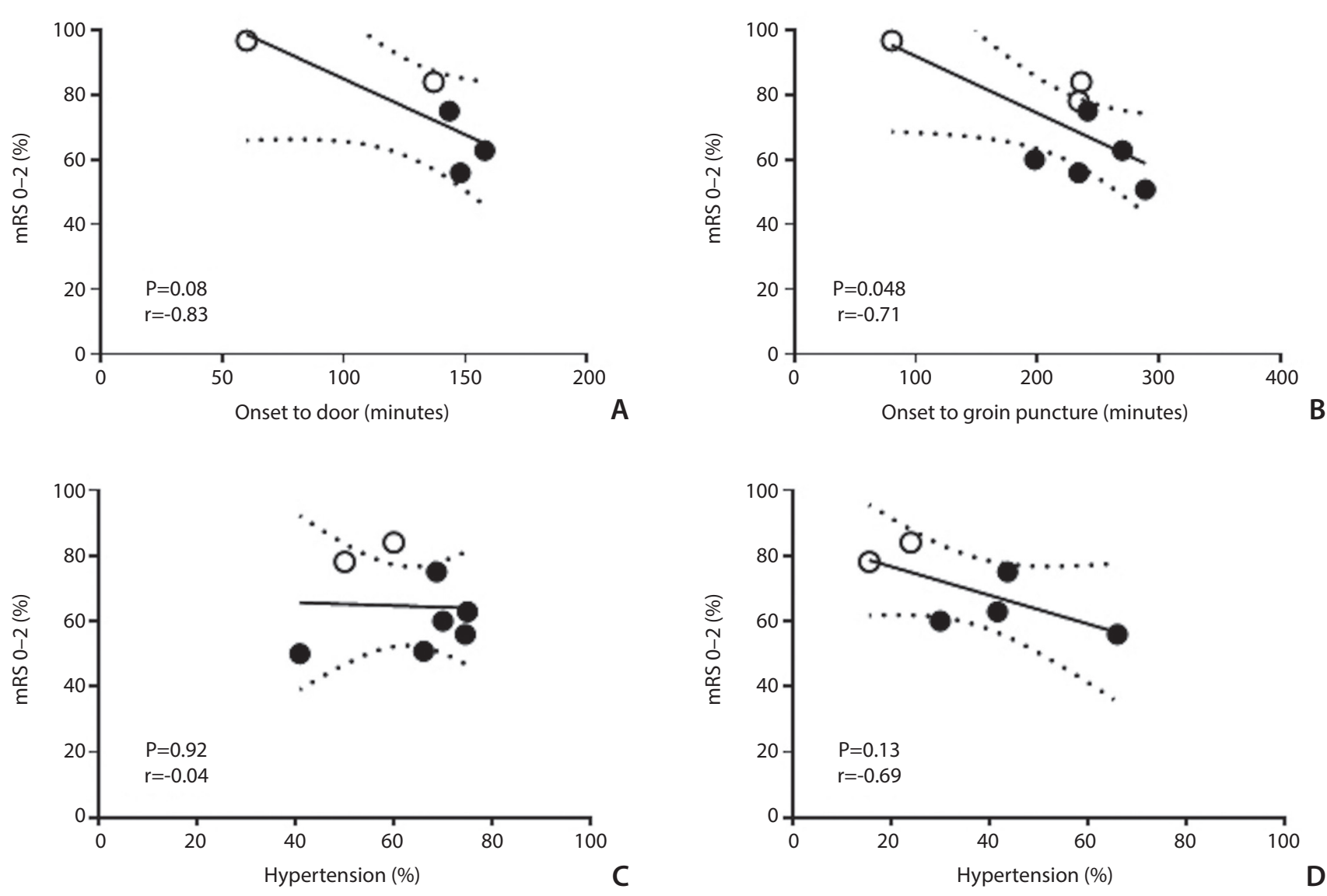

Fig. 5. Correlation analysis for good clinical outcome mRS 0-2. Open circles represent contact aspiration, closed circles represent stent-retriever thrombectomy. mRS, modified Rankin scale.

tact aspiration techniques were demonstrated in distal M2 acute ischemic strokes. In particular, favorable recanalization rates and thrombectomy procedure times were equivalent between the two cohorts. The proportion of patients with good mRS outcome was similar between the two thrombectomy techniques; however, excellent clinical outcome at 90-days was significantly lower for stent-retrieval thrombectomy, although this was on a background of higher baseline comorbidities of hypertension and hyperlipidemia as well as increased symptom onset to groin puncture times. Our analysis also demonstrated that onset to groin puncture time was the best correlate of clinical outcome at follow-up and justifies the differences observed in clinical outcomes between the aspiration and stent-retrieval groups in our meta-analysis. As such, our findings suggest that endovascular treatment of MCA M2 occlusions by contact aspiration is at least similar to that of stent-retrieval, and that reducing onset to groin puncture time is associated with improved 90-day clinical outcome. Overall, our findings share similarities with level 1 evidence for endovascular therapy for large anterior vessel occlusion strokes, supporting the evolving role of endovascular thrombectomy for distal occlusions.

To date, endovascular therapy in distal MCA strokes remains controversial for several reasons. Firstly, distal MCA strokes tend to have more favorable presentations and outcomes relative to large vessel occlusions, due to smaller infarct and penumbra volumes. In such patients, alteplase tends to have reasonable effectiveness and thus not all patients are candidates for intra-arterial intervention. ${ }^{8,9}$ However, recanalization with alteplase may be inadequate for some patients, and endovascular intervention could be of benefit. In a natural history study of acute ischemic strokes, Hernández-Pérez et al. ${ }^{31}$ demonstrated poor clinical outcomes (mRS $>2$ at 3 months) and 3\% deaths in distal MCA occlusion cases without reperfusion therapy. Lima et al. ${ }^{32}$ observed poor $m R S>2$ in $46 \%$ of 48 cases of acute M2 occlusions, resulting in $21 \%$ mortality when intervention was not performed. As such, there is certainly a subset of distal occlusion acute 
ischemic stroke patients who could benefit from a more aggressive endovascular therapy as first line treatment. With regards to current randomized evidence thus far, distal MCA occlusions such as M2 were significantly under-represented in the major randomized trials proving the benefit of endovascular therapy for large vessel occlusion strokes. Of the randomized studies included in the HERMES collaborative meta-analysis, ${ }^{6}$ only two trials ${ }^{1,2}$ enrolled a minor fraction of cases with distal occlusions. Of five trials assessed, 7.3\% of patients had isolated M2 segment occlusions, and 51 of these cases received endovascular treatment. Their analysis was unable to confirm any harm of endovascular therapy in distal MCA occlusions, ${ }^{6}$ suggesting that the benefits of endovascular therapy in acute ischemic strokes extends to the M2 occlusion group of stroke patients. However, the nature of the M2 occlusions which could have been "large" M2 and not "distal M2" is unclear, and may have been considered M1 by local means and $\mathrm{M} 2$ by core lab, given that not all trials were core-lab adjudicated.

With regards to the choice of thrombectomy technique, there are concerns regarding their complication profiles. The smaller, thinned-walled vasculature of distal MCA occlusions are susceptible to perforation or dissection complications of thrombectomy, particularly for stent-retrievers. Contact aspiration has been an increasingly popular option for mechanical thrombectomy, particularly with recent advancements in catheter technology resulting in increased suction force, flexibility, and less traumatic tips. ${ }^{11,20}$ However, there are currently no randomized trials comparing aspiration versus stent-retriever techniques for distal MCA M2 occlusions. The recently published ASTER (The Contact Aspiration vs. Stent Retriever for Successful Revascularization) study compared 192 patients who underwent first-line contact aspiration versus 189 cases of first-line stent retriever cases in patients with acute ischemic strokes. The proportion of cases with successful revascularization, change in NIHSS score, and adverse event rates were comparable between the two arms of this trial; however, it was noted that M2 occlusions comprised only $20.7 \%$ of patients in this trial, and hence their conclusions cannot be directly extrapolated to all distal MCA strokes. Their results were similar to those of the present meta-analysis, in that there was a similar rate of distal embolization, perforation, and dissection complications between the two techniques. In terms of technical success rate in our meta-analysis, both aspiration first approaches and stent-retriever techniques achieved equivalent reperfusion rates, indicating similar technical efficacy of either approach.

In agreement with prior studies, such as the ASTER randomized trial, that did not demonstrate any significant difference in clinical outcomes between aspiration versus stent-retriever approaches, our meta-analysis demonstrated similar TICI 2b-3 recanalization rates for both thrombectomy techniques. While mRS 0-1 was significantly more favorable for aspiration, this difference was not necessarily due to differences in the efficacy of treatment, but could also be potentially attributed to confounders, such as differences in baseline characteristics or time delays to groin puncture. Correlation analysis demonstrated that onset to groin puncture time was significantly negatively correlated with clinical outcome. As such, the differences in 90-day favorable mRS in aspiration versus stent-retriever cohorts may be explained by a larger portion of aspiration first cases having a shorter duration of onset to puncture, thus resulting in improved clinical outcomes. This is supported by the multivariate adjusted analysis of Kim et al. ${ }^{9}$, which demonstrated that time to recanalization was an independent predictor of favorable clinical outcome for M2 segment occlusions. However, in contrast to Kim et al. ${ }^{9}$, the present meta-analysis did not find age to be a significant confounding factor. The relative benefits of contact aspiration versus stent-retrieval on clinical outcomes at follow-up require further investigation and confirmation by prospective multi-center randomized controlled trials.

The present study is limited by several constraints. First, there was no control medical therapy group or natural history cohort to compare the endovascular therapy outcomes to. The HERMES study subgroup analysis demonstrated no significant difference in outcome between medically managed and endovascular intervened cases in terms of good mRS outcome, with an odds ratio of 1.28 (0.51-3.21), however raw data for this subgroup was not provided and thus could not be included for comparative analysis in the present meta-analysis. In terms of endpoints, several key parameters such as time of onset to imaging or time of imaging to groin puncture were not reported by the majority of included studies and their influence on clinical outcome could not be assessed in the present meta-analysis. A variety of stent-retrieval devices and aspiration catheters were used, which further adds heterogeneity to the pooled analysis. Varying anatomical complexity, such as branching and tortuosity, could not be accounted for in this meta-analysis and has the potential to contribute to the reduced effectiveness of mechanical thrombectomy. There was also a lack of ho- 
mogeneity in terms of definition of $\mathrm{M} 2$ used in the included studies (Supplementary Table 2), and there is prior evidence that M2 segment definition and occlusion location can lead to differences in reported revascularization rates and good outcomes. ${ }^{29}$ The use of general anesthesia versus conscious sedation has also been shown to have a significant influence on clinical outcome in large vessel occlusions; however, we were unable to test this in our meta-analysis. Mokin et al. ${ }^{24}$ reported however that choice of sedation, whether conscious or general, had no effect on the outcomes of thrombectomy for $\mathrm{M} 2$ occlusions. We were also unable to perform a meta-analysis of adjusted effect sizes for various confounders, as in most cases this was not reported in the included studies. Other limitations which undermine the validity of the results include the retrospective observational nature of the majority of included studies, which lends itself to selection bias. Furthermore, this study is not randomized, and the difference in baseline characteristics, such as time of symptom onset to groin puncture, complicates comprehensive analysis of this data. Finally, there was a moderate to strong heterogeneity in many of the analyses we performed, which is probably due to the many differences in definitions, methods, and study populations included in this meta-analysis.

\section{CONCLUSION}

In conclusion, both endovascular techniques achieved recanalization rates greater than $80 \%$ and 90 -day outcomes of minimal disability with similar complication rates. The current literature is skewed by aspiration cases being performed sooner after onset of stroke compared to stent-retriever cases, and this limits interpretation and generalizability of pooled outcomes. The current findings need to be assessed in the setting of a prospective, multi-center randomized trial.

\section{SUPPLEMENTARY MATERIALS}

Supplementary materials related to this article can be found online at https://doi.org/10.5469/neuroint.2018.00997.

\section{REFERENCES}

1. Berkhemer OA, Fransen PS, Beumer D, van den Berg LA, Lingsma HF, Yoo AJ, et al. A randomized trial of intraarterial treatment for acute ischemic stroke. NEng/ J Med 2015;372:11-20

2. Campbell BC, Mitchell PJ, Kleinig TJ, Dewey HM, Churilov L, Yassi $\mathrm{N}$, et al. Endovascular therapy for ischemic stroke with perfusion-imaging selection. N Engl J Med 2015;372:1009-1018

3. Goyal M, Demchuk AM, Menon BK, Eesa M, Rempel JL, Thornton J, et al. Randomized assessment of rapid endovascular treatment of ischemic stroke. N Engl J Med 2015;372:1019-1030

4. Jovin TG, Chamorro A, Cobo E, de Miquel MA, Molina CA, Rovira $A$, et al. Thrombectomy within 8 hours after symptom onset in ischemic stroke. N Engl J Med 2015;372:2296-2306

5. Saver JL, Goyal M, Bonafe A, Diener HC, Levy El, Pereira VM, et al. Stent-retriever thrombectomy after intravenous t-PA vs. t-PA alone in stroke. N Engl J Med 2015;372:2285-2295

6. Goyal M, Menon BK, van Zwam WH, Dippel DW, Mitchell PJ, Demchuk AM, et al. Endovascular thrombectomy after large-vessel ischaemic stroke: a meta-analysis of individual patient data from five randomised trials. Lancet 2016;387:17231731

7. Phan K, Zhao DF, Phan S, Huo YR, Mobbs RJ, Rao PJ, et al. Endovascular therapy including thrombectomy for acute ischemic stroke: a systematic review and meta-analysis with trial sequential analysis. J Clin Neurosci 2016;29:38-45

8. Dorn F, Lockau H, Stetefeld H, Kabbasch C, Kraus B, Dohmen C, et al. Mechanical thrombectomy of M2-occlusion. J Stroke Cerebrovasc Dis 2015;24:1465-1470

9. Kim YW, Son S, Kang DH, Hwang YH, Kim YS. Endovascular thrombectomy for M2 occlusions: comparison between forced arterial suction thrombectomy and stent retriever thrombectomy. J Neurointerv Surg 2017;9:626-630

10. Powers WJ, Derdeyn CP, Biller J, Coffey CS, Hoh BL, Jauch EC, et al. 2015 American Heart Association/American Stroke Association focused update of the 2013 guidelines for the early management of patients with acute ischemic stroke regarding endovascular treatment: a guideline for healthcare professionals from the American Heart Association/American Stroke Association. Stroke 2015;46:3020-3035

11. Phan K, Dmytriw AA, Teng I, Moore JM, Griessenauer C, Ogilvy $C$, et al. A direct aspiration first pass technique vs standard endovascular therapy for acute stroke: a systematic review and meta-analysis. Neurosurgery 2018;83:19-28

12. Turk AS, Frei D, Fiorella D, Mocco J, Baxter B, Siddiqui A, et al. ADAPT FAST study: a direct aspiration first pass technique for 
acute stroke thrombectomy. J Neurointerv Surg 2014;6:260-264

13. Turk AS, Spiotta A, Frei D, Mocco J, Baxter B, Fiorella D, et al. Initial clinical experience with the ADAPT technique: a direct aspiration first pass technique for stroke thrombectomy. J Neurointerv Surgery 2014;6:231-237

14. Turk AS, Turner R, Spiotta A, Vargas J, Holmstedt C, Ozark S, et al. Comparison of endovascular treatment approaches for acute ischemic stroke: cost effectiveness, technical success, and clinical outcomes. J Neurointerv Surg 2015;7:666-670

15. Chen CJ, Wang C, Buell TJ, Ding D, Raper DM, Ironside N, et al. Endovascular mechanical thrombectomy for acute middle cerebral artery M2 segment occlusion: a systematic review. World Neurosurg 2017;107:684-691

16. Moher D, Liberati A, Tetzlaff J, Altman DG; PRISMA Group. Preferred reporting items for systematic reviews and meta-analyses: the PRISMA statement. PLoS Med 2009;6:e1000097

17. Higashida RT, Furlan AJ, Roberts $H$, Tomsick T, Connors B, Barr J, et al. Trial design and reporting standards for intra-arterial cerebral thrombolysis for acute ischemic stroke. Stroke 2003;34:e109-e137

18. Hozo SP, Djulbegovic B, Hozo I. Estimating the mean and variance from the median, range, and the size of a sample. BMC Med Res Methodol 2005;5:13

19. Higgins JP, Thompson SG. Quantifying heterogeneity in a meta-analysis. Stat Med 2002;21:1539-1558

20. Altenbernd J, Kuhnt O, Hennigs S, Hilker R, Loehr C. Frontline ADAPT therapy to treat patients with symptomatic M2 and M3 occlusions in acute ischemic stroke: initial experience with the penumbra ACE and 3MAX reperfusion system. J Neurointerv Surg 2017;10:434-439

21. Lapergue B, Blanc R, Gory B, Labreuche J, Duhamel A, Marnat G, et al. Effect of endovascular contact aspiration vs stent retriever on revascularization in patients with acute ischemic stroke and large vessel occlusion: the ASTER randomized clinical trial. JAMA 2017;318:443-452

22. Coutinho JM, Liebeskind DS, Slater LA, Nogueira RG, Baxter BW, Levy El, et al. Mechanical thrombectomy for isolated M2 occlusions: a post hoc analysis of the STAR, SWIFT, and SWIFT PRIME studies. AJNR Am J Neuroradiol 2016;37:667-672
23. Flores A, Tomasello A, Cardona P, de Miquel MA, Gomis M, Garcia Bermejo P, et al. Endovascular treatment for M2 occlusions in the era of stentrievers: a descriptive multicenter experience. $J$ Neurointerv Surg 2015;7:234-237

24. Mokin M, Primiani CT, Ren Z, Kan P, Duckworth E, Turner RD 4th, et al. Endovascular treatment of middle cerebral artery M2 occlusion strokes: clinical and procedural predictors of outcomes. Neurosurgery 2017;81:795-802

25. Park JS, Kwak HS. Manual aspiration thrombectomy using penumbra catheter in patients with acute $\mathrm{M} 2$ occlusion : a single-center analysis. J Korean Neurosurg Soc 2016;59:352-356

26. Protto S, Sillanpää N, Pienimäki JP, Matkaselkä I, Seppänen J, Numminen H. Stent retriever thrombectomy in different thrombus locations of anterior cerebral circulation. Cardiovasc Intervent Radio/ 2016;39:988-993

27. Salahuddin H, Ramaiah G, Slawski DE, Shawver J, Buehler M, Zaidi SF, et al. Mechanical thrombectomy of M1 and M2 middle cerebral artery occlusions. J Neurointerv Surg 2018;10:330-334

28. Sarraj A, Sangha N, Hussain MS, Wisco D, Vora N, Elijovich L, et al. Endovascular therapy for acute ischemic stroke with occlusion of the middle cerebral artery M2 segment. JAMA Neurol 2016;73:1291-1296

29. TomsickTA, Carrozzella J, Foster L, Hill MD, von Kummer R, Goyal $M$, et al. Endovascular therapy of M2 occlusion in IMS III: role of M2 segment definition and location on clinical and revascularization outcomes. AJNR Am J Neuroradiol 2017;38:84-89

30. Vargas J, Spiotta AM, Fargen K, Turner RD, Chaudry I, Turk A. Experience with a direct aspiration first pass technique (ADAPT) for thrombectomy in distal cerebral artery occlusions causing acute ischemic stroke. World Neurosurg 2017;99:31-36

31. Hernández-Pérez M, Pérez de la Ossa N, Aleu A, Millán M, Gomis M, Dorado L, et al. Natural history of acute stroke due to occlusion of the middle cerebral artery and intracranial internal carotid artery. J Neuroimaging 2014;24:354-358

32. Lima FO, Furie $K L$, Silva GS, Lev MH, Camargo EC, Singhal $A B$, et al. Prognosis of untreated strokes due to anterior circulation proximal intracranial arterial occlusions detected by use of computed tomography angiography. JAMA Neurol 2014;71:151157 